Research Article

Genetics of Microorganisms

\title{
Transcriptome sequencing and screening of genes related to glucose availability in Schizosaccharomyces pombe by RNA-seq analysis
}

\author{
Çağatay Tarhan ${ }^{1}$ (D) and Özgür Çakır ${ }^{1}$ (D) \\ ${ }^{I}$ Istanbul University, Faculty of Science, Department of Molecular Biology and Genetics, Istanbul, Turkey.
}

\begin{abstract}
While calorie restriction is the most used experimental intervention to increase lifespan in numerous model organisms, increasing evidence suggests that excess glucose leads to decreased lifespan in various organisms. To fully understand the molecular basis of the pro-aging effect of glucose, it is still important to discover genetic interactions, gene expression patterns, and molecular responses depending on glucose availability. Here, we compared the gene expression profiles in Schizosaccharomyces pombe mid-log-phase cells grown in three different Synthetic Dextrose media with $3 \%, 5 \%$, and $8 \%$ glucose, using the RNA sequencing method. Expression patterns of genes that function in carbohydrate metabolism were downregulated as expected, and these genes were downregulated in line with the increase in glucose content. Significant and consistent changes in the expression were observed such as genes that encoding retrotransposable elements, heat shock proteins, glutathione S-transferase, cell agglutination protein, and conserved fungal proteins. We group some genes that function together in the transcription process and mitotic regulation, which have recently been associated with glucose availability. Our results shed light on the relationship between excess glucose, diverse cellular processes, and aging.
\end{abstract}

Keywords: Glucose availability, Schizosaccharomyces pombe, aging, gene regulation, RNA-Seq.

Received: July 16, 2020; Accepted: June 01, 2021.

\section{Introduction}

The nutritional condition is one of the most important determinants of cellular and organismal life-span. It has been shown in a wide range of species that, while overnutrition accelerates aging, nutritional restriction, which is also called calorie restriction, delays it (Reverter-Branchat et al., 2004). Once it was realized that limited nutrition not only increases longevity but prevents or retards some age-related diseases (Anderson and Weindruch, 2012), the focus of aging studies has shifted to uncover the underlying genetic and molecular mechanisms of calorie restriction and aging. Mainly two approaches are used to understand these mechanisms. One of them, single-gene mutations or overexpression, has been used to identify the genes whose overexpression or deletion mimic the effect of calorie restriction, or that have a considerable effect on longevity by some other mechanisms. In this regard, although they mostly consider the protein coding regions and exclude the role of noncoding and regulatory regions, many attempts have been made in a variety of organisms such as Escherichia coli, Saccharomyces cerevisiae, Drosophila melanogaster, Caenorhabditis elegans and mice (Fontana et al., 2010, Rallis et al., 2013, Dancy et al., 2014, Sideri et al., 2014, Boehm et al., 2016).

Another approach is to perform whole-genome expression profiling when cells or organisms are grown in poor or rich nutritional conditions, or when they are in the natural aging process (Rallis et al., 2014, Baumgart et al., 2016, Hall et al., 2017).

Send correspondence to Çağatay Tarhan. Department of Molecular Biology and Genetics, Faculty of Science, Istanbul University, 34134 Vezneciler, Istanbul, Turkey. E-mail: cagatay.tarhan@ istanbul.edu.tr.
This method is quite fruitful because it promises to gain more integrative insights and to find new genes, interactions or regulatory mechanisms that contribute to the process of natural aging or adaptation to an experimental condition.

Both approaches have revealed important insights into the relationship between nutrition and some well-known fundamental cellular pathways, such as insulin/insulin-like growth factor 1 (IGF-1) signaling (IIS), the Target of Rapamycin (TOR) and the energy-sensing pathway AMP-activated protein kinase (AMPK). Their downstream elements and interactions with other cellular processes have also been identified. For example, it is well established in various organisms that the IIS pathway is activated in response to nutrients and acts in part through mTOR to impact lifespan (Papadopoli et al., 2019). On the other hand, amino acid metabolism, oxygen, various stressors or energy input affect mTOR activity and thereby regulates other metabolic pathways that control cell growth. Activation of the mTOR pathway leads to the inhibition of autophagy, which is an important cellular self-degradative process in the determination of cell fate, aging, or disease development. AMPK is activated by changes in the cellular AMP/ATP ratio and the increasing activity has been shown to extend lifespan in various organisms (Mair et al., 2011, Wierman et al., 2017).

Since glucose is the common source of energy in most prokaryotes and eukaryotes, its availability directly affects these and other pathways thereby organismal aging. Manipulating the glucose concentration in food or media, many researchers have shown alteration and/or restructuring in the activity of these pathways and the expression levels of their members. For example, elevated glucose in diet shortened lifespan in C. elegans by downregulating proteins such as AMPK, FOXO, and glyoxalase (Lee et al., 2009) In S. cerevisiae, glucose 
limiting leads to $35 \%$ extension in lifespan (Lin et al., 2000), and lowering the glucose concentration from $2 \%$ to $0.1 \%$ prolonged yeast lifespan up to $50 \%$ (Jiang et al., 2000). On the other hand, increasing glucose concentration in the medium from $2 \%$ to $10 \%$ induced superoxide ion production, which causes the inhibition of growth arrest and led to DNA replication stress in $S$. cerevisiae (Weinberger et al., 2010). After determining that glucose shortened lifespan in a dosedependent manner in S. pombe, Roux et al. (2009) showed that not only the glucose metabolism but also the extracellular glucose signaling might be responsible for decreasing chronological lifespan. They found that the deletion of git $3^{+}$, which encodes a $\mathrm{G}$ protein-coupled receptor protein that senses environmental glucose, causes an effect that mimics calorie restriction (Roux et al., 2009). Chen and Runge (2009) suggested that glucose shortens the lifespan of $S$. pombe cells partly because remaining glucose signals cells to grow and thereby prevents them to be arrested in a quiescent state. Besides, they showed that the deletion of serine/threonine-specific protein kinase (AKT) orthologs $s c k 1^{+}$and $s c k 2^{+}$, caused a significant lifespan extension especially in the presence of excess glucose. This finding suggests that these genes efficiently function in the control of longevity (Chen and Runge, 2009). Although it is not well established that glucose accelerates aging in humans and other mammals, some studies have made significant contributions to answering this question. For example, excess glucose consumption increased the percentage of polyploid $\beta$-cells, and this increase may reflect premature aging of these $\beta$-cells in mice (White et al., 1985). More recently, Mortuza et al. (2013) reported that excess glucose accelerates aging in different types of human endothelial cells by decreased activity of sirtuins which are mediated through FOXO1. In another study, Zhang et al. (2017) showed that mesenchymal stem cells aging-induced when the cells treated with 11 or $22 \mathrm{mM}$ glucose for 14 days, and they suggested that the Akt/mTOR signaling pathway acts as the primary mediator in the aging process induced by excess glucose (Zhang et al., 2017). These and other attempts have yielded important insights into the role of glucose and energy metabolism in the process of aging. Yet there is still more to discover by testing different conditions and media, investigating different time intervals, using active substances, mutant strains or experimental combinations in the manipulation of the aging process of an organism.

In this study, to evaluate the transcriptome-wide changes in the presence of excess glucose, we performed a genomewide mRNA expression profiling (RNA-seq) analysis in wildtype Schizosaccharomyces pombe which has become a popular model organism in aging studies in recent years. We used three Synthetic Dextrose (SD) media which contain 3\%, 5\%, and $8 \%$ glucose, represented by $\mathrm{T} 1, \mathrm{~T} 2$, and $\mathrm{T} 3$ respectively. As glucose concentration increased, the expression of genes associated with carbohydrate metabolism was downregulated as expected. We observed a marked and consistent change in the expression of the genes encoding retrotransposable elements, heat shock proteins, glutathione S-transferase, cell agglutination protein, as well as genes encoding conserved fungal proteins. Besides, we evaluated the changes in the expression of a group of genes (e.g. transcription coactivators or mitotic progression regulators) that have recently been associated with glucose availability and metabolism. Our results contribute to correlate diverse cellular processes with excess glucose availability and its pro-aging effects.

\section{Material and Methods}

\section{Yeast strain, media, and growth conditions}

The wild type strain of Schizosaccharomyces pombe $\left(972 \mathrm{~h}^{-}\right)$was used in all experiments. Because it was reported that the Synthetic Dextrose (SD) media were an appropriate condition for lifespan experiments and cells grown in SD with excess glucose showed the evolutionarily conserved response to lifespan (Chen and Runge, 2009), we used this medium. Cells from a single colony were inoculated in three different SD media with $3 \%, 5 \%$, and $8 \%$ glucose, which are represented by $\mathrm{T} 1, \mathrm{~T} 2$, and $\mathrm{T} 3$ respectively. Initial cell density was $5 \times 10^{4}$ cells $/ \mathrm{ml}$ and cells were grown up to mid-log phase in $25 \mathrm{ml}$ of medium in a $100 \mathrm{ml}$ flask at $30^{\circ} \mathrm{C}$ with orbital shaking at $180 \mathrm{rpm}$.

\section{Total RNA isolation}

Total RNAs were isolated from mid-log cells using GeneJET RNA Purification Kit (Thermo Fisher Scientific) following the manufacturer's instructions for each sample. The purity and quantity of RNAs were measured using a Nanodrop 2000 spectrophotometer (Nanodrop Technologies, USA). Total RNAs were then used in further analysis. Three replicates were used for each sample and replicates were then pooled for sequencing according to their concentrations.

\section{Library preparation and sequencing}

Fragmentation, adapter binding, cDNA library preparation, and RNA sequencing were performed by Beijing Genome Institute (Shenzhen, China). Briefly, the RNAs were fragmented in fragmentation buffer. After this treatment, cDNA synthesis was done by using the fragments as templates with random hexamer primers. Adapters were ligated to short fragments, then, PCR was performed. Lastly, Illumina HiSeq 2000 was used for paired end sequencing.

\section{Bioinformatic analysis of sequence data}

Firstly, the raw reads of low quality were eliminated before any advanced analysis. Filtering was done to eliminate adapter sequences (SOAPnuke software, ver. 1.5.6, http://soap. genomics.org.cn/), sequences with a high content of unknown bases more than $10 \%$ and sequences with low-quality scores (less than 10). Residual reads were accepted as clean reads. Clean reads were then aligned to the Schizosaccharomyces pombe genome and genes using BWA (Burrows-Wheeler Aligner, ver 0.7.12, (Li and Durbin, 2010)) and BOWTIE (ver.1.1.2) (Langmead et al., 2009) software, respectively. 
The expression of aligned genes and isoforms was then measured and quantified by RSEM software package (ver. 1.2.25) (Li and Dewey, 2011). RSEM takes into account the maximum likelihood abundance estimates by the ExpectationMaximization (EM) algorithm as its statistical model, including the modeling of paired-end (PE) and variable-length reads, fragment length distributions, and quality scores, to specify the transcripts that are isoforms of the same gene. The expression levels were calculated by using the FPKM (fragments per kilobase per million reads) and Tophat method was used to determine the alternative splicing event detection (2.1.0) (Trapnell et al., 2009). We did not want to determine a random FPKM value to determine whether a transcript was expressed. However, analysis of all transcripts with expression levels greater than zero must include FPKM values very close to zero. Therefore, we chose 0.005 FPKM as the lower limit for the subsequent analysis. Differentially expressed genes were screened according to the Poisson distribution method and NOIseq package (FDR $<0.001$ and $\log 2$ [sample1/sample2] $>1$ ) (ver. 2.14.1) (Tarazona et al., 2011). For gene ontology analysis, differentially expressed genes first mapped to GO terms in gene ontology database (www.geneontology.org). The gene numbers for each $\mathrm{GO}$ term were calculated (Corrected $\mathrm{p}$ value $\leq 0.05$ as threshold). The mapped genes were classified according to three different categories, molecular function, cellular component, and biological process. The figures are drawn with respect to significantly enriched GO terms. The functional classification was done by using WEGO software (Ye et al., 2006). Lastly, KEGG was used to analyze significantly enriched pathways between samples (Qvalue $\leq 0.05$ ) (Kanehisa et al., 2008). KEGG enrichment analysis was done by the algoritm developed by BGI. Data were submitted to https:// www.ncbi.nlm.nih.gov/geo/ (Accession number GSE159723).

\section{Results}

The RNAs from the cells grown in SD medium with $3 \%, 5 \%$, and $8 \%$ glucose were sequenced on Illumina Hiseq 2000 platform. A total of 74,654,458 raw reads were obtained from the sequencing of three different libraries. Quality control was done by filtering the raw data (Figure 1). The adapter sequences and the sequences which contain more than $10 \%$ unknown bases and low-quality score sequences were eliminated. The number for clean reads was 73,082,162. While genome mapping rates were $94.83,94.85$ and 94.51 , gene mapping rates were $90.28,90.92$ and $89.72 \%$ for T1, T2 and T3 samples, respectively (Table 1). Slightly over 5000 genes were found to be expressed in each sample. For T1, $78.50 \%$ of the reads were perfectly matched with the reference genome while $76.20 \%$ of the reads were matched perfectly to reference genes. The perfect matches on a reference genome for T2 and T3 were 79.10 and 79.24 respectively. Also, perfect matches on reference genes for $\mathrm{T} 2$ and $\mathrm{T} 3$ were 77.13 and 76.47 respectively. The statistics of sequencing were shown in Table 1.
To investigate the effect of different glucose concentrations on gene expression profile, we analyzed the T1, T2, and T3 samples with respect to differentially expressed genes (Figure 2). The number of commonly expressed genes and only the number of genes whose expression significantly differentiated were considered and given for each condition. Accordingly, 5061 genes were expressed commonly in both $\mathrm{T} 1$ and T2 samples, while only 8 genes were expressed in $\mathrm{T} 1$ and 21 genes in $\mathrm{T} 2$. When we compared the T1 and $\mathrm{T} 3,5045$ were in common, 24 were expressed in $\mathrm{T} 1$ and 13 were expressed in T3. Lastly, 5049 were expressed in both T2 and T3 while 33 were expressed only in T2 and 9 in T3. Glucose concentration significantly affected gene expression between samples. When we compared T1 and T3, 104 genes were downregulated $(\mathrm{FDR} \leq 0.001, \log 2$ ratio $\leq 1)$ and 205 genes were found to be upregulated (FDR $\leq 0.001$, $\log 2$ ratio $\geq 1$ ) (Figure 3 ). For T2 and T1 comparison, there were 54 genes downregulated $(\mathrm{FDR} \leq 0.001, \log 2$ ratio $\leq 1)$ and 8 genes upregulated (FDR $\leq 0.001, \log 2$ ratio $\geq 1$ ). The upregulated genes were $80(\mathrm{FDR} \leq 0.001, \log 2$ ratio $\geq 1)$ and downregulated ones were $101(\mathrm{FDR} \leq 0.001, \log 2$ ratio $\leq 1)$ when T2 and T3 were compared.

After gene ontology annotations to reveal the distribution of gene functions, functional classification was performed for differentially expressed genes. The gene ontology analysis showed that the metabolic process (GO ID: 0008152, number of genes: 38 ) and cellular process (GO ID: 0009987, number of genes: 31) were the categories with the highest gene numbers in biological process in the comparison between T1 and T3. In the cellular component class in the same comparison, cell (GO ID: 0005623, number of genes: 21) and cell part (GO ID: 0044464, number of genes: 21) categories had the highest gene numbers. Lastly, in molecular function, binding (GO ID: 0005488, number of genes: 39 ) and catalytic activity (GO ID: 0003824, number of genes: 48) were prominent.

For the comparison between $\mathrm{T} 2$ and $\mathrm{T} 1$, localization (GO ID: 0051179, number of genes: 5), metabolic process (GO ID: 0008152, number of genes: 7) for biological process class, cell (GO ID: 0005623, number of genes: 2), cell part (GO ID: 0044464, number of genes: 2) and membrane (GO ID: 0016020, number of genes: 3) for cellular component class, binding (GO ID: 0005488, number of genes: 6) and catalytic activity (GO ID: 0003824, number of genes: 10) for molecular function class had the highest gene numbers.

For the comparison between $\mathrm{T} 2$ and $\mathrm{T} 3$, cellular process (GO ID: 0009987, number of genes: 13), metabolic process (GO ID: 0008152, number of genes: 23) for biological process class, cell (GO ID: 0005623, number of genes: 5), cell part (GO ID: 0044464, number of genes: 5), membrane part (GO ID: 0044425, number of genes: 5) and membrane (GO ID: 0016020, numbero f genes: 8) for cellular component class, binding (GO ID: 0005488, number of genes: 21 ) and catalytic activity (GO ID: 0003824, number of genes: 34 ) for molecular function class had the highest gene numbers (Figure 4). Also, 
Classification of Raw Reads (T1)

Reads of (T1) contains:

1. N $(9382,0.04 \%)$

2. low qual $(107022,0.43 \%)$

3. Adapter $(430344,1.73 \%)$

4. clean reads $(24337956,97.8 \%)$

A

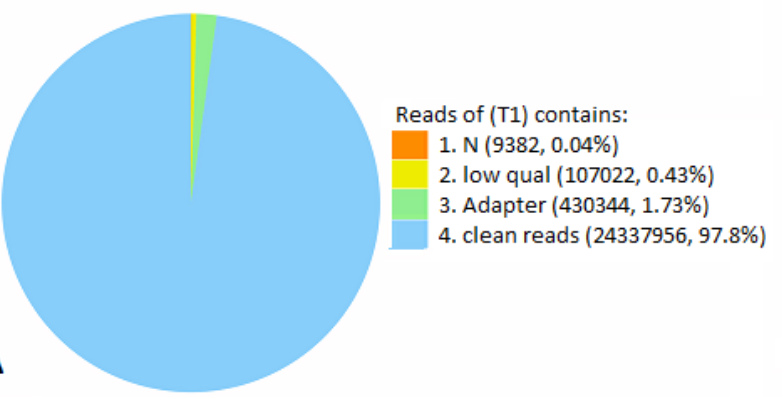

B

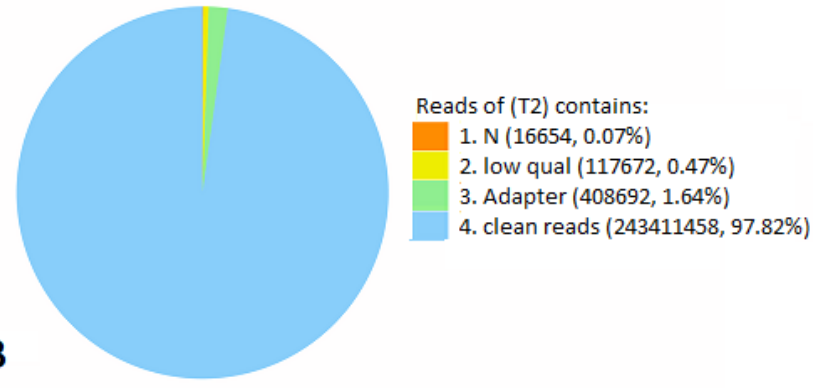

Classification of Raw Reads (T3)

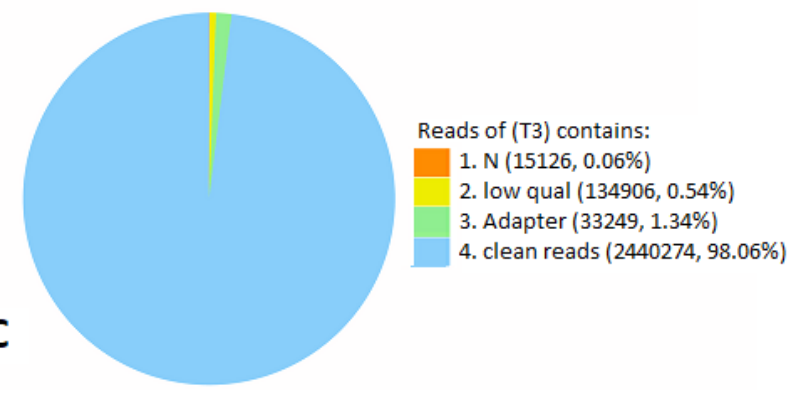

Figure 1 - Classification of raw reads in samples A) T1, B) T2, C) T3.

Table 1 - Statistics of raw data belonging to different samples.

\begin{tabular}{|c|c|c|c|c|c|c|}
\hline & Map to Genome & Reads Number & Percent & Map to Gene & Reads Number & Percent \\
\hline \multirow{8}{*}{$\mathrm{T} 1$} & Total Reads & $24,337,956$ & $100.00 \%$ & Total Reads & $24,337,956$ & $100.00 \%$ \\
\hline & Total BasePairs & $2,433,795,600$ & $100.00 \%$ & Total BasePairs & $2,433,795,600$ & $100.00 \%$ \\
\hline & Total Mapped Reads & $23,080,889$ & $94.83 \%$ & Total Mapped Reads & $21,971,788$ & $90.28 \%$ \\
\hline & Perfect Match & $19,104,654$ & $78.50 \%$ & Perfect Match & $18,544,717$ & $76.20 \%$ \\
\hline & Mismatch & $3,976,235$ & $16.34 \%$ & Mismatch & $3,427,071$ & $14.08 \%$ \\
\hline & Unique Match & $22,343,264$ & $91.80 \%$ & Unique Match & $18,848,762$ & $77.45 \%$ \\
\hline & Multi-position Match & 737,625 & $3.03 \%$ & Multi-position Match & $3,123,026$ & $12.83 \%$ \\
\hline & Total Unmapped Reads & $1,257,067$ & $5.17 \%$ & Total Unmapped Reads & $2,366,166$ & $9.72 \%$ \\
\hline \multirow{8}{*}{$\mathrm{T} 2$} & Total Reads & $24,341,458$ & $100.00 \%$ & Total Reads & $24,341,458$ & $100.00 \%$ \\
\hline & Total BasePairs & $2,434,145,800$ & $100.00 \%$ & Total BasePairs & $2,434,145,800$ & $100.00 \%$ \\
\hline & Total Mapped Reads & $23,086,706$ & $94.85 \%$ & Total Mapped Reads & $22,130,242$ & $90.92 \%$ \\
\hline & Perfect Match & $19,233,184$ & $79.01 \%$ & Perfect Match & $18,775,050$ & $77.13 \%$ \\
\hline & Mismatch & $3,853,522$ & $15.83 \%$ & Mismatch & $3,355,187$ & $13.78 \%$ \\
\hline & Unique Match & $22,362,920$ & $91.87 \%$ & Unique Match & $19,150,654$ & $78.68 \%$ \\
\hline & Multi-position Match & 723,786 & $2.97 \%$ & Multi-position Match & $2,979,588$ & $12.24 \%$ \\
\hline & Total Unmapped Reads & $1,254,752$ & $5.15 \%$ & Total Unmapped Reads & $2,211,214$ & $9.08 \%$ \\
\hline \multirow{8}{*}{ T3 } & Total Reads & $24,402,748$ & $100.00 \%$ & Total Reads & $24,402,748$ & $100.00 \%$ \\
\hline & Total BasePairs & $2,440,274,800$ & $100.00 \%$ & Total BasePairs & $2,440,274,800$ & $100.00 \%$ \\
\hline & Total Mapped Reads & $23,062,621$ & $94.51 \%$ & Total Mapped Reads & $21,895,314$ & $89.72 \%$ \\
\hline & Perfect Match & $19,336,002$ & $79.24 \%$ & Perfect Match & $18,660,950$ & $76.47 \%$ \\
\hline & Mismatch & $3,726,619$ & $15.27 \%$ & Mismatch & $3,234,364$ & $13.25 \%$ \\
\hline & Unique Match & $22,080,264$ & $90.48 \%$ & Unique Match & $18,925,418$ & $77.55 \%$ \\
\hline & Multi-position Match & 982,357 & $4.03 \%$ & Multi-position Match & $2,969,896$ & $12.17 \%$ \\
\hline & Total Unmapped Reads & $1,340,127$ & $5.49 \%$ & Total Unmapped Reads & $2,507,432$ & $10.28 \%$ \\
\hline
\end{tabular}



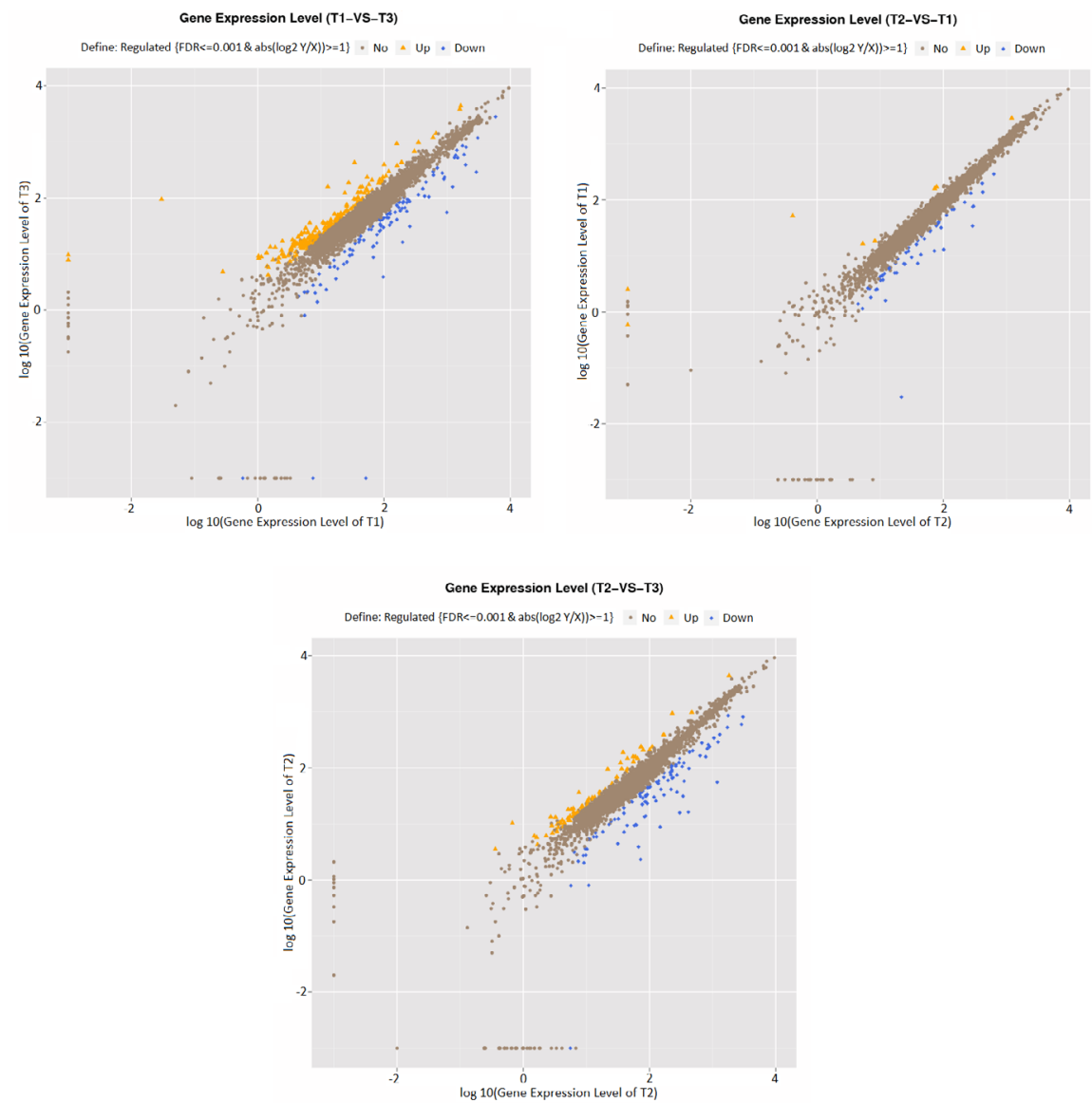

Figure 2 - Differential expression levels of T1, T2, and T3 samples. Yellow and blue color represent differentially expressed genes, Brown color represents the genes whose expression does not differ in samples.

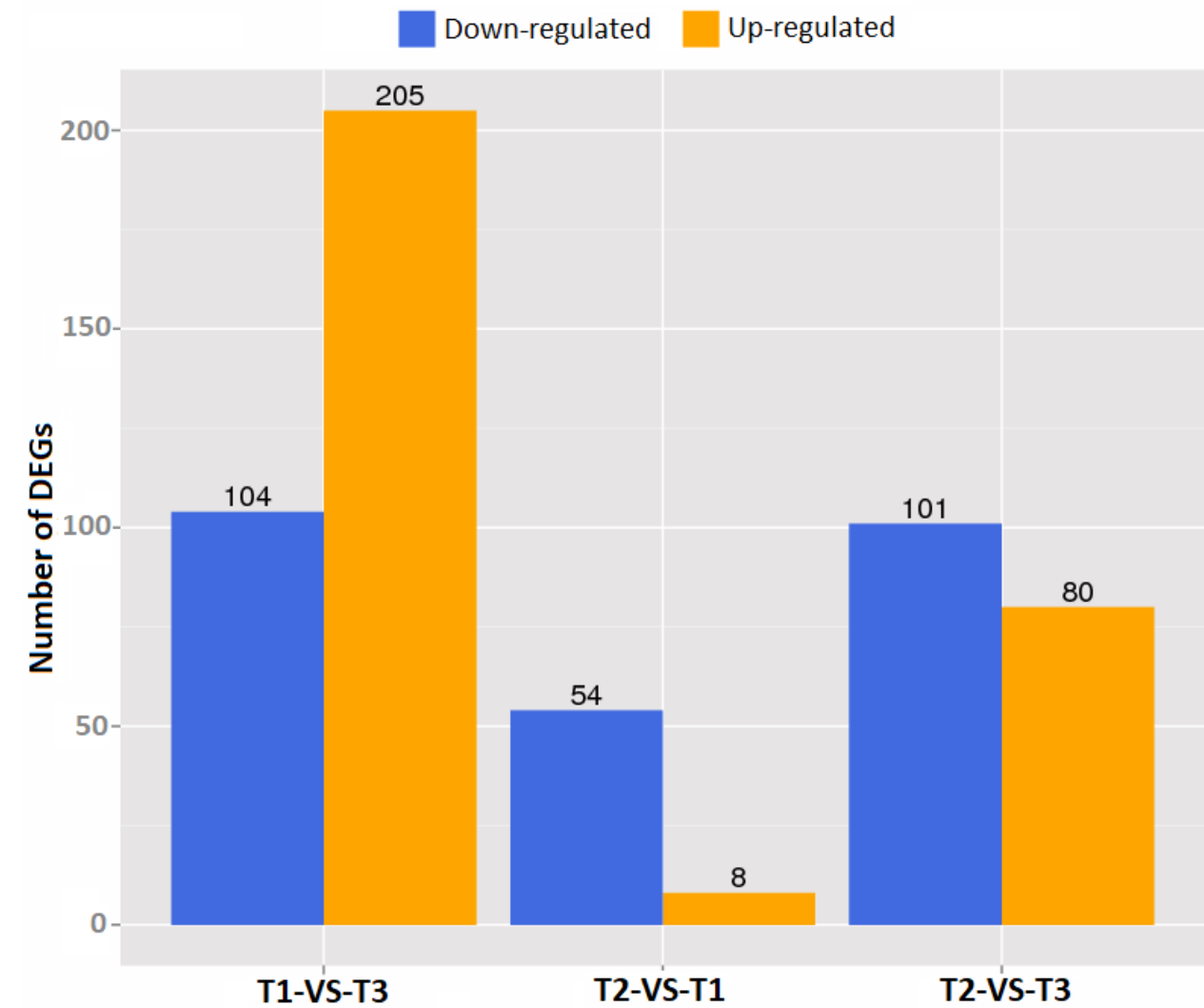

Figure 3 - The gene numbers of differentially expressed genes. Blue color represents downregulation and yellow color represents upregulation. 

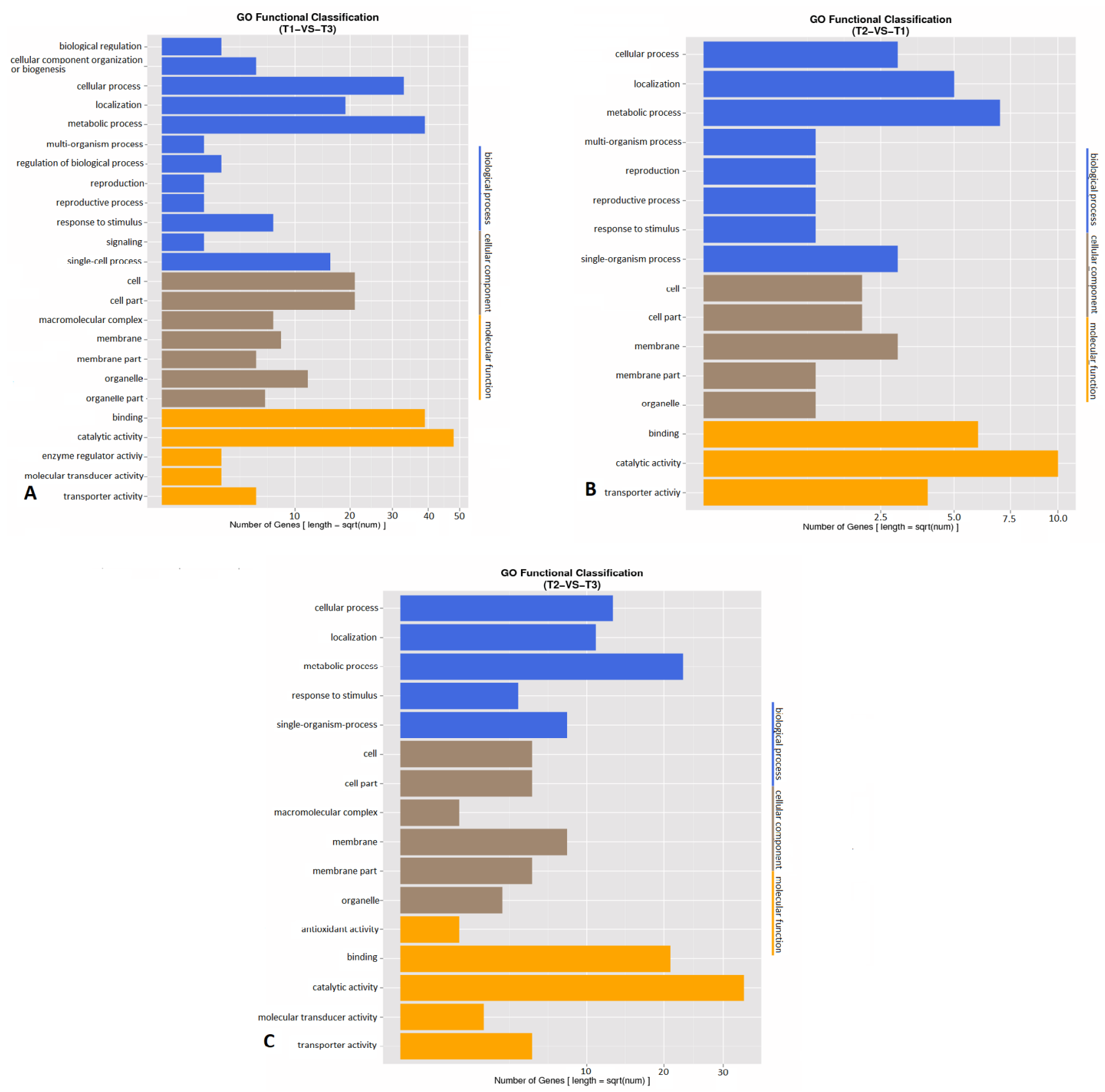

Figure 4 - GO classification of differentially expressed genes between groups, A) T1 vs T3, B) T2 vs T1, C) T2 vs T3.

KEGG pathway enrichment analysis was done for differentially expressed genes between samples and the top 20 pathways are shown in Figure 5.

The quantitative change in the expression of individual genes depending on the glucose concentration in the medium is given in Table $\mathrm{S} 1$.

\section{Discussion}

\section{Gene functions in carbohydrate metabolism}

$\mathrm{gall}^{+}, \mathrm{gall}^{+}$, and $\mathrm{gallO}^{+}$genes function in galactose utilization in $S$. pombe. Although the growth of $S$. pombe does not require galactose indispensably, galactosylation is crucial for some cellular processes including the maintenance of normal cell shape, drug tolerance, and nonsexual flocculation. (Takegawa and Matsuzawa, 2014) To do this, at first galactose is transported into the cytosol and then the two enzymes,
Gall and Gal7, involves in the conversion of galactose into UDP-galactose. Under glucose-limited conditions, Gal10 functions especially in galactosylation of cell-surface proteins (Matsuzawa et al. , 2011). In our study, the expressions of gall $^{+}$, gall $^{+}$gall $^{+}$are downregulated at elevated glucose levels. These results were consistent with the other studies. For example, it is reported that while the expression of gall $10^{+}$is strongly upregulated by glucose repression, gal10 $0^{+}$ is repressed in glucose-rich medium in S. pombe (Suzuki et al., 2010). In S. cerevisiae, glucose repression of GAL genes occurs through Mig1p global repressor protein (Nehlin et al., 1991). Besides, the transcription level of SPBPB2B2.11 which is predicted to encode a nucleotide-sugar 4,6-dehydratase enzyme was downregulated in the presence of $8 \%$ glucose when compare to SD with 5\% glucose. This gene is located very close to the GAL genes and the expression pattern is 

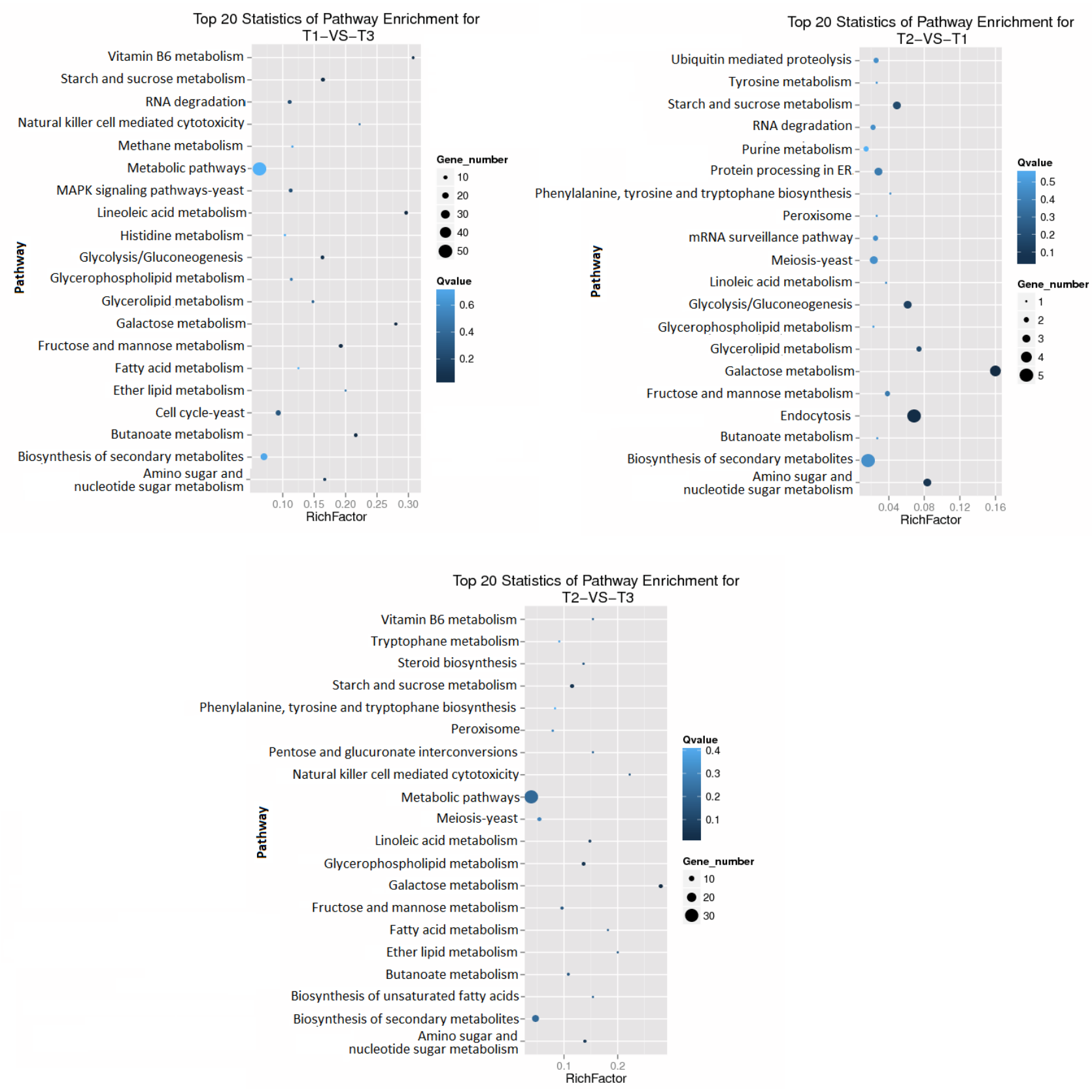

Figure 5 - Chart of top 20 pathways between different treatment groups.

similar to those of these genes regarding galactose presence in the culture medium (Matsuzawa et al., 2011).

The transcription level of invertase encoding $i n v l^{+}$and mitochondrial glycerol dehydrogenase encoding $g l d l^{+}$are also decreased significantly under glucose-rich conditions. It has been reported that the expression of these genes is downregulated when a high level of glucose is present in the culture medium. The fission yeast transcriptional regulator Scrl mediates the repression of $i n v l^{+}$and $g l d l^{+}$ (Tanaka et al., 1998, Matsuzawa et al., 2010). Similarly, the expression level of $g u t 2^{+}$which encodes the glycerol3 -phosphate dehydrogenase decreased consistently when glucose concentration was high. Although a direct link has not been shown between glucose availability and the gut $2^{+}$ expression in $S$. pombe, Saccharomyces cerevisiae ortholog of $g u t 2^{+}$was shown to be subjected to glucose repression (Grauslund and Ronnow, 2000).

S. pombe has eight genes that encode the corresponding eight hexose transporters $\left(g h t 1^{+}\right.$to $g h t 8^{+}$) (Heiland et al., 2000). According to our results, two of them, ght $1^{+}$and $g h t 5^{+}$are subjected to glucose repression in both conditions. Kim et al., (2010) showed that all eight transporter genes are nonessential when the glucose concentration was 3\% in culture medium (Kim et al., 2010). It is also reported that the transcript level of the major hexose transporter $g h t 5^{+}$and $g h t 1^{+}$, which has a moderate affinity to glucose, was increased in the low-glucose medium. In the same study, it was also demonstrated that when the glucose concentration high, the $g h t 5^{+}$expression was repressed by Scr1 which also represses inv $1^{+}$transcription (Saitoh et al., 2015). S. pombe genome 
encodes another Scr1-like protein, called Rsv1. This protein was shown to act as a transcriptional repressor and the deletion of $r s v 1^{+}$induced the genes that are involved in carbohydrate metabolism including $g h t 1^{+}$(Mata et al., 2007). In our study, when the glucose concentration in the medium was $8 \%$, the expression level of $r s v I^{+}$was 2,82 fold lower than that observed in SD with 5\%. Taken together, our results showed that the expression levels of genes that function in carbohydrate metabolism consistently changed and regulated according to glucose content in the medium. When Vassiliadis and others performed a transcriptome analysis of preferential glucose utilization in $S$. pombe, they revealed that carbon metabolism pathways significantly upregulated in the glucose-deficient condition and they clarified that Scr1p regulates certain genes (e.g. inv $1+$, ght $5+$, and $g l d 1+$ ) involved in carbon metabolism, hexose uptake, gluconeogenesis, TCA cycle. However, in that study, Yeast Extract Supplemented (YES) medium was used (Vassiliadis et al., 2019). Another genome-wide study, which also used YES as the medium, found that carbohydrate metabolism and meiotic differentiation were repressed in glucose rich medium (Malecki et al., 2016). Considering the similar results we obtained, our study also contributed to prove that SD medium could be used in longevity and energy metalobism related issues.

gid $2^{+}$is predicted to be the subunit of glucose-induced degradation deficient (GID) complex ubiquitin-protein ligase E3. In gluconeogenesis, fructose-1,6-bisphosphatase is expressed when there is no fermentable carbon source in medium, but in the presence of glucose, the enzyme undergoes a rapid degradation (Holzer, 1989). In S. cerevisiae, Gid2/Rmd5 is the $S$. pombe gid2 ${ }^{+}$ortholog and responsible for the proteasomal degradation of the enzyme to block gluconeogenesis (Santt et al., 2008). Thus, the increase in gid $2^{+}$expression in the $\mathrm{T} 3$ condition may indicate a similar regulation in $S$. pombe.

agll+ functions in maltose utilization when especially carbon source in the medium is switched from glucose to maltose or glucose is completely depleted (Kato et al., 2013). In this study, its expression was downregulated in all tested conditions, implying that cells in that phase might still be using glucose and there was no need to induce agll+ expression.

\section{Genes other than those that function in carbohydrate metabolism}

While the increase in the expression of one of the two Tf2-type retrotransposable elements, SPAPB15E9.03c, in T2/T1 was 9,71 fold, the expression of SPAC26A3.13c upregulated 2,24 fold in T3 condition when compared to T2 condition. Although Tf2 mRNA levels are not necessarily accompanied by a proportional change in mobilization (Murton et al., 2016), this expression pattern suggests an association between glucose availability and $T f 2$ expression levels. On the other hand, Mallet et al. (2017) showed that antisense Tf2 transcripts were maximally induced during meiotic division cycle, especially when sexual differentiation program was initiated. According to our results, the expression of mat $1-M c$ gene, which encodes a mating-type m specific polypeptide and controls the mating-type (Kelly et al., 1988) was markedly lower (-8.72 fold) in T2 condition when compared to T1.
This change in the expression might have indicated that the sexual differentiation and some other dependent process halted in the presence of excess carbon source. It has been reported that the expression of this gene activated by Ste11p (Sugimoto et al., 1991) and it was shown in another study that starvation induces the ste $11^{+}$transcript through decreased Protein Kinase A (PKA) activity (Valbuena and Moreno, 2010). On the other hand, it was shown that aforementioned Scr1p is required to repress sexual differentiation under glucose-sufficient conditions (Vassiliadis et al., 2019) Thus, it seems that, in the presence of excess glucose, activated PKA activity may have downregulated the ste $11^{+}$expression and, in turn, mat 1 $M c$ expression. This downregulation may cause a decrease in Tf2 antisense transcript production and this may lead to a marked SPAPB15E9.03c upregulation. This mechanism, in the reverse direction, can also explain the SPAC26A3.13c upregulation. On the other hand, the expression pattern of these retrotransposable elements, which are positively correlated with glucose availability, could suggest an answer about the relationship between retrotransposon expression and aging. It is well documented that there is a strong correlation between retrotransposition activity, genome instability, and aging and that the expression of many types of retrotransposons increases with age in a wide variety of organisms including yeast, Drosophila, and mammals (De Cecco et al., 2013, Li et al., 2013, Hu et al., 2014). However, whether this increase in expression is a consequence of aging or is directly involved in the aging process is not clear (Maxwell, 2016). As seen in many other organisms, calorie restriction extends $S$. pombe chronological lifespan when, for example, glucose levels were decreased 3 or 40 fold (Chen and Runge, 2009). In our study, RNAs were isolated from young mid-log phase cells. Thus, in concordance with the glucose availability, the elevated retrotransposon expression in young cells may directly contribute and determine the aging process under the condition of high glucose before these cells enter the stationary phase.

Glutathione S-transferases are a group of enzymes that catalyze the conjugation of glutathione with many exogenous and endogenous substances in the binding and detoxification of toxic compounds. In $S$. pombe, three different glutathione S-transferase coding genes, gst $1^{+}$, gst $2^{+}$, and $g s t 3^{+}$, were identified (Veal et al., 2002). According to Kim et al., among them, only the expression of gst $3+$ was regulated by glucose or sucrose availability and its mRNA level was higher when a low concentration of glucose was present (Kim et al., 2004). They inferred that, as seen in $S$. cerevisiae, glucose depletion causes an increase in reactive oxygen species and this triggers gst $3^{+}$ expression. We observed that gst $2^{+}$expression induced when glucose concentration was increased. Thus gst $2^{+}$expression may also be regulated by glucose availability and induced when glucose concentration is increased.

hsp16+ is a small heat shock protein whose mRNA level is known to be induced by heat stress and its expression is activated through the transcription factor atfl $1^{+}$(Taricani et al., 2001). It was suggested that $h s p 16^{+}$expression may be controlled by nutritional conditions (Danjoh and Fujiyama, 1999). Indeed, the expression of HSP26 (Saccharomyces cerevisiae ortholog of $h s p 16^{+}$), increased when glucose levels were low (Amoros and Estruch, 2001). In our study, the 
expression of $h s p 16^{+}$was downregulated consistently when the glucose concentration increased from both $3 \%$ and $5 \%$ to $8 \%$. It was shown that the $h s p 16^{+}$expression repressed by $S$. pombe HIRA proteins which are histone chaperones that mediate nucleosome assembly and the activation of these proteins are also regulated by atf1 $1^{+}$(Greenall et al., 2006). It is well known that glucose starvation is one of the environmental stresses and it seems that, under glucose-rich condition, the $h s p 16^{+}$ expression is not necessary and suppressed by a mechanism which possibly involves atf $1^{+}$. In our study, like that of $h s p 16^{+}$, the expression of $s s a l^{+}$(or $h s p 70^{+}$) which encodes another predicted heat shock protein was downregulated consistently when the glucose concentration increased. Similarly, it was reported that hsp70 mRNA levels in rat hepatocytes were significantly higher in caloric-restricted conditions than those observed when there was no such restriction (Heydari et al., 1993). On the other hand, it has long been known that Hsp70 counteracts protein aggregation along with Hsp104 and the association of Hsp104/Hsp70 is essential in longevity (Glover and Lindquist, 1998). In our study, the expression of hsp104 was downregulated when glucose level higher. Taken together, consistent downregulation of these heat shock proteins may cause a loss of cellular proteostasis which is considered as a hallmark of the aging process. Thus, excess sugar may deteriorate the cellular protective mechanism and make them less active just as seen during aging. Indeed, overexpression of these molecular chaperones ameliorates the age-associated decline in proteostasis and extends lifespan in Caenorhabditis and Drosophila (Walker and Lithgow, 2003, Morrow et al., 2004).

$\mathrm{mam}^{+}$encodes an M-type-specific cell surface adhesion protein for conjugation between sexually flocculating cells (Mata and Bahler, 2006). Although the transcription of mam $^{+}{ }^{+}$is induced under nitrogen starvation (Seike et al., 2013) and it is activated by mat1-Mc recruited Ste11, its expression seemed independent from mat1-Mc in our study. We observed that mam $^{+}$expression increased in both $\mathrm{T} 2$ and $\mathrm{T} 3$ conditions when compared to $\mathrm{T} 1$ condition while mat1-Mc expression downregulated in the $\mathrm{T} 1$ condition. This result raises the possibility of another function for $\mathrm{mam}^{+}$other than adhesion. On the other hand, according to Liu et. al. (2015) flocculation activity in wild type $S$. pombe decreased with the lowering of glucose concentration. This result may support our finding that $\mathrm{mam}^{+}$expression upregulated in response to elevated glucose concentrations but further studies are needed to clarify this situation.

When comparing the gene expression profile in $\mathrm{T} 3$ condition to those that $\mathrm{T} 1$, we saw that the expression of a group of genes related especially to kinetochore assembly, chromosome segregation, and mitotic progression markedly increased. These genes were $n b l 1^{+}, a p c 13^{+}, s p c 19^{+}, t e a 2^{+}, m i s 6^{+}$, mis $18^{+}$, mis $15^{+}$. Mis proteins function as a structural core and regulator of the kinetochore (Muller and Almouzni, 2017). Together with some other subunits, Mis 18 complex is required for the centromeric localization of the Mis6-Mis15 complex and Cnp1 during the cell cycle (Takahashi et al., 2005). Nbl1 is a borealin homolog gene and a subunit of a chromosome passenger complex (CPC) which is required to destabilize and repair inaccurate kinetochore attachments. Binding of borealin to nucleosome is essential for this complex to associate with the chromosome and for the error-free chromosome segregation (Abad et al., 2019). Apc13 is a member of the Anaphase Promoting Complex (APC) which maintains genomic stability and initiates the transition from metaphase to anaphase and coordinate chromosome segregation in mitosis by routing specific proteins for degradation (Zhang et al., 2016). Spc19 is a component of the DASH complex which forms a ring around a microtubule and responsible for the correct chromosome segregation and bipolar attachment of sister chromatids on the mitotic spindle (Sanchez-Perez et al., 2005). Tea2 encodes a kinesin that regulates microtubule growth by transporting regulatory factors to the growing microtubule tips (Busch et al., 2004). Taken together, the increase in the expression of these genes may indicate a cellular activity that provides errorfree chromosome segregation. Glucose detection results in the activation of the cAMP-dependent protein kinase A (PKA) in $S$. pombe and PKA coordinates cell proliferation depending on glucose status (Hoffman, 2005). Although many studies suggest that PKA is an inhibitor of the APC, the interaction between the cAMP-PKA pathway and the kinetochore is thought to be more complex (Ma et al., 2012). On the other hand, it is thought that the APC may be involved in enhancing genomic stability through repairing DNA damage that occurs during chromosome segregation but this process has not been fully elucidated (Harkness, 2018). Recent works revealed a connection between glucose availability and kinetochore activity and bipolar spindle formation during mitosis (Shah et al., 2019, Tanabe et al., 2020). Accordingly, higher glucose levels destabilize kinetochore attachment and weaken kinetochore-microtubule binding through phosphorylation of Dam1 which is $S$. cerevisiae ortholog of Spc19. Growth defects and monopolar spindle formation in a kinesin-5 mutant was partially rescued by $\mathrm{pkal}^{+}$deletion or glucose limitation in $S$. pombe. Under glucose-rich condition, cells may tolerate higher rates of mitotic infidelity to reproduce faster. On the other hand, the expression of these mitotic regulators may have increased to cope with the genome instability resulting, for example, from increased retrotransposon activity due to the presence of excess glucose. The relationship between nutrient availability and mitotic regulators has only recently been studied. Thus, further investigations are needed to clarify this link.

The other group whose expression in $\mathrm{T} 3$ condition at least two-fold increased when compared to T1 includes three transcription regulators or coactivators. One of them, spt $3^{+}$ is a subunit of the SAGA (Spt-Ada-Gcn5 acetyltransferase) complex which acts as a general cofactor, chromatin remodeler, and sets the accessibility for DNA transcription (Helmlinger et al., 2008). taf $^{+}$is predicted to be a subunit of TFIID, a complex composed of TATA-binding protein (TBP) and TBP associated factors (TAFs) (Matangkasombut et al., 2004) The third one, med $13^{+}$is the component of Cdk8 module of the multiprotein Mediator complex which is a central integrator of transcription. In addition to the targeting of CDK 8 by several different intracellular signaling pathways, the loss of Med13 may lead to the early entry of the cell into mitosis, suggesting that this protein also regulates the mitotic progression (Banyai et al., 2017). On the other hand, the components of TFIID, SAGA complex, and Mediator are known to interact with 
each other in certain cellular processes, but there is much unknown about this relationship (Donczew et al., 2020, Papai et al., 2020). It is reported that the SAGA complex is a direct target of nutrient-sensing pathways and regulates, for example, switching from proliferation to sexual differentiation when the glucose level is low (Helmlinger et al., 2008, Laboucarie et al., 2017). On the other hand, the deletion of the SPT3 gene was shown to lead to a severe decrease in Ty1 retrotransposon transcription in S. cerevisiae (Winston et al., 1984, Curcio et al., 2015). Similarly, the marked increase in the retrotransposable element expression depending on elevated glucose levels that we observed in this study may be explained partly by the increase in $s p t 3^{+}$expression. Interestingly, overexpression of MED13 improved glucose tolerance and insulin sensitivity in mice cardiomyocytes (Dannappel et al., 2018). This effect of MED13 results from increased energy expenditure and regulation of numerous genes involved in energy balance (Grueter et al., 2012). Besides, the knockdown of MED13 increases susceptibility to obesity by regulating Wingless in Drosophila (Lee et al., 2014). Thus, the induced expression of med13 in this study may contribute to regulating energy balance in the presence of excess glucose. Taken together, our findings are consistent with these results and may help to clarify the link between glucose availability and the coactivators.
The putative interaction map of proteins that encoded by all genes whose expression were significantly changed acording to glucose availability was shown in Figure 6.

Finally, the expression of a group of genes that can be classified unassigned, sequence orphan, dubious, or conserved fungal and whose functions are unknown were also downregulated or upregulated in response to elevated glucose levels. Importantly, while the expression of SPBC660.05 was significantly reduced, SPAC959.06c was upregulated in response to increasing glucose concentration. These genes are predicted to be conserved fungal proteins and because the consistent change in their expression suggests a role in glucose metabolism they can be the candidates for further studies.

\section{Conclusions}

Besides being a universal energy source, glucose also functions as a signal molecule in cells. Therefore, it affects many cellular processes such as division, proliferation, and aging. From this point of view, we conducted a genome-wide analysis to clarify how glucose presence affects genome-wide gene regulation. Our results indicated that excess glucose, as expected, downregulated many genes that function in carbohydrate metabolism. On the other hand, we found that there were significant and consistent changes in the

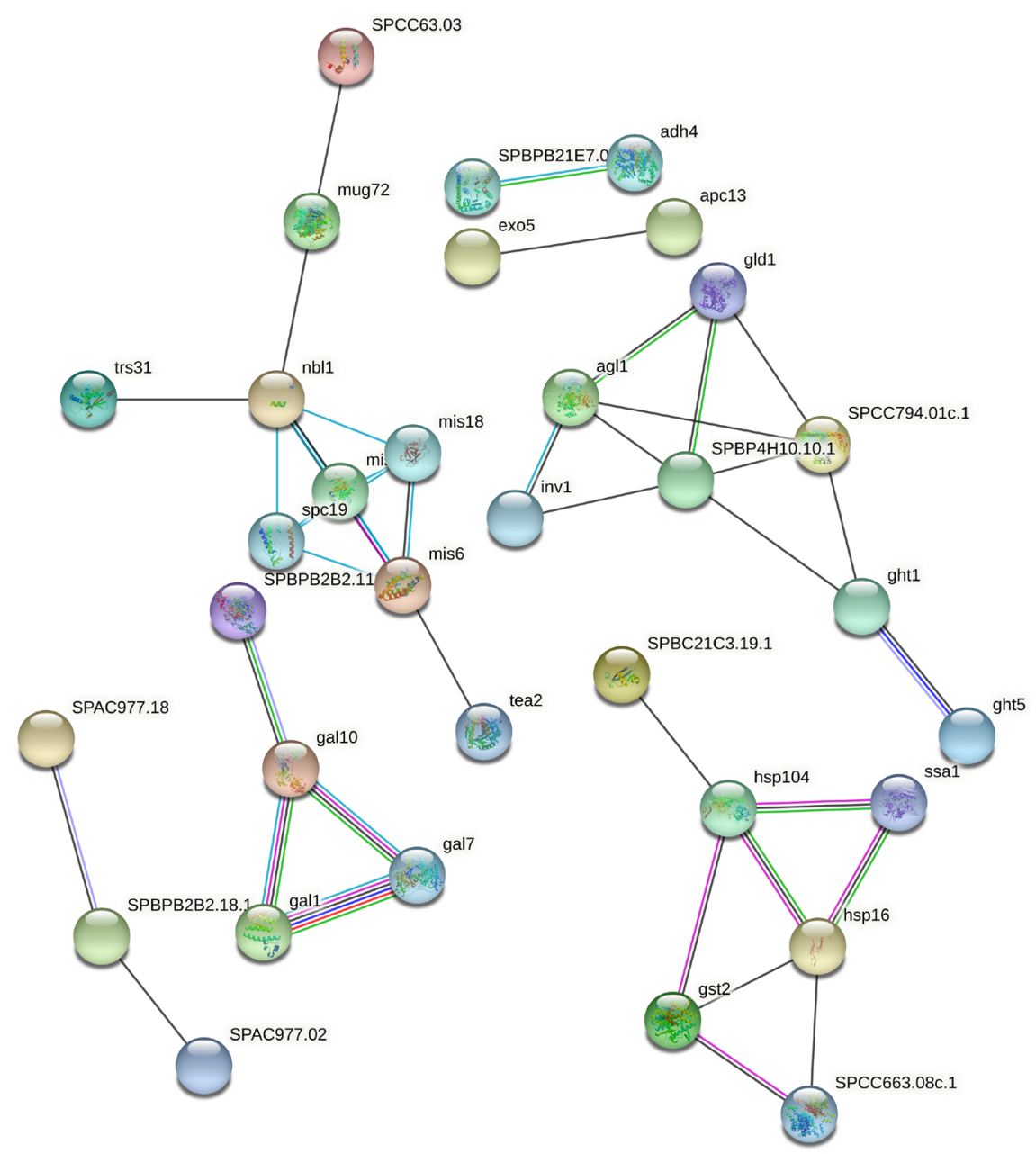

Figure 6 - The putative interaction map of proteins that encoded by all genes whose expression were significantly changed acording to glucose availability. Network was created through STRING website (ver. 11.0). Interaction sources included experiments, databases, co-expression, neighborhood, gene fusion and co-ocurrence. Minimum required interaction score was set as 0.400 . Detailed explanation is given in the text. 
expression of genes encoding retrotransposons, heat shock proteins, transcription regulators, and proteins that provide genomic stability. We suggest some complementary roles and possible interactions of these gene expression changes that can explain the possible pro-aging effect of excess glucose. These suggestions may contribute to the findings obtained in recent years regarding the relationship between glucose availability and diverse cellular mechanisms. We also observed significant changes in the expression of some genes that have not yet been well characterized in $S$. pombe. Thus, their roles in glucose metabolism and glucose-dependent cellular response can be the subject of future studies. We hope that the large scale analysis we have performed will serve as the basis for more detailed studies on the relationship between glucose availability and aging.

\section{Acknowledgements}

This research was supported by the Istanbul University Research Fund, project no. 51562

\section{Conflict of Interest}

The authors declare that there is no conflict of interest that could be perceived as prejudicial to the impartiality of the reported research.

\section{Authors Contributions}

ÇT conceived the study; ÇT conducted the experiments; ÖÇ analyzed the data; ÇT and ÖÇ wrote the manuscript.

\section{References}

Abad MA, Ruppert JG, Buzuk L, Wear M, Zou J, Webb KM, Kelly DA, Voigt P, Rappsilber J, Earnshaw WC et al. (2019) Borealinnucleosome interaction secures chromosome association of the chromosomal passenger complex. J Cell Biol 218:3912-3925.

Amoros M and Estruch F (2001) Hsflp and Msn2/4p cooperate in the expression of Saccharomyces cerevisiae genes HSP26 and HSP104 in a gene- and stress type-dependent manner. Mol Microbiol 39:1523-3152.

Anderson RM and Weindruch R (2012) The caloric restriction paradigm: implications for healthy human aging. Am J Hum Biol 24:101-106.

Banyai G, Szilagyi Z, Baraznenok V, Khorosjutina $\mathrm{O}$ and Gustafsson CM (2017) Cyclin C influences the timing of mitosis in fission yeast. Mol Biol Cell 28:1738-1744.

Baumgart M, Priebe S, Groth M, Hartmann N, Menzel U, Pandolfini L, Koch P, Felder M, Ristow M, Englert C et al. (2016) Longitudinal RNA-Seq analysis of vertebrate Aging identifies Mitochondrial Complex I as a small-molecule-sensitive modifier of lifespan. Cell Syst 2:122-132.

Boehm A, Arnoldini M, Bergmiller T, Roosli T, Bigosch C and Ackermann M (2016) Genetic manipulation of glycogen allocation affects replicative lifespan in E. coli. PLoS Genet 12:e1005974.

Busch KE, Hayles J, Nurse P and Brunner D (2004) Tea2p kinesin is involved in spatial microtubule organization by transporting tiplp on microtubules. Dev Cell 6:831-843.

Chen BR and Runge KW (2009) A new Schizosaccharomyces pombe chronological lifespan assay reveals that caloric restriction promotes efficient cell cycle exit and extends longevity. Exp Gerontol 44:493-502.
Curcio MJ, Lutz S and Lesage P (2015) The Ty1 LTR-Retrotransposon of budding yeast, Saccharomyces cerevisiae. Microbiol Spectr 3: MDNA3-0053-2014.

Dancy BM, Sedensky MM and Morgan PG (2014) Effects of the mitochondrial respiratory chain on longevity in C. elegans. Exp Gerontol 56:245-255.

Danjoh I and Fujiyama A (1999) Ras-mediated signaling pathway regulates the expression of a low-molecular-weight heat-shock protein in fission yeast. Gene 236:347-52.

Dannappel MV, Sooraj D, Loh JJ and Firestein R (2018) Molecular and in vivo functions of the CDK8 and CDK19 kinase modules. Front Cell Dev Biol 6:171.

De Cecco M, Criscione SW, Peterson AL, Neretti N, Sedivy JM and Kreiling JA (2013) Transposable elements become active and mobile in the genomes of aging mammalian somatic tissues. Aging (Albany NY) 5:867-883.

Donczew R, Warfield L, Pacheco D, Erijman A and Hahn S (2020) Two roles for the yeast transcription coactivator SAGA and a set of genes redundantly regulated by TFIID and SAGA. Elife 9:e50109.

Fontana L, Partridge L and Longo VD (2010) Extending healthy life span--from yeast to humans. Science 328:321-326.

Glover JR and Lindquist S (1998) Hsp104, Hsp70, and Hsp40: a novel chaperone system that rescues previously aggregated proteins. Cell 94:73-82.

Grauslund M and Ronnow B (2000) Carbon source-dependent transcriptional regulation of the mitochondrial glycerol-3phosphate dehydrogenase gene, GUT2, from Saccharomyces cerevisiae. Can J Microbiol 46:1096-100.

Greenall A, Williams ES, Martin KA, Palmer JM, Gray J, Liu C and Whitehall SK. (2006) Hip3 interacts with the HIRA proteins Hip1 and Slm9 and is required for transcriptional silencing and accurate chromosome segregation. J Biol Chem 281:8732-8739.

Grueter CE, van Rooij E, Johnson BA, DeLeon SM, Sutherland LB, Qi X, Gautron L, Elmquist JK, Bassel-Duby R and Olson EN (2012) A cardiac microRNA governs systemic energy homeostasis by regulation of MED13. Cell 149:671-683.

Hall H, Medina P, Cooper DA, Escobedo SE, Rounds J, Brennan KJ, Vincent C, Miura P, Doerge R and Weake VM (2017) Transcriptome profiling of aging Drosophila photoreceptors reveals gene expression trends that correlate with visual senescence. BMC Genomics 18:894.

Harkness TAA (2018) Activating the Anaphase Promoting Complex to enhance genomic stability and prolong lifespan. Int J Mol Sci 19:1888.

Heiland S, Radovanovic N, Hofer M, Winderickx J and Lichtenberg H (2000) Multiple hexose transporters of Schizosaccharomyces pombe. J Bacteriol 182:2153-2162.

Helmlinger D, Marguerat S, Villen J, Gygi SP, Bahler J and Winston F (2008) The $S$. pombe SAGA complex controls the switch from proliferation to sexual differentiation through the opposing roles of its subunits Gen5 and Spt8. Genes Dev 22:3184-3195.

Heydari AR, Wu B, Takahashi R, Strong R and Richardson A(1993) Expression of heat shock protein 70 is altered by age and diet at the level of transcription. Mol Cell Biol 13:2909-2918.

Hoffman CS (2005) Glucose sensing via the protein kinase A pathway in Schizosaccharomyces pombe. Biochem Soc Trans 33:257-260.

Holzer H (1989) Proteolytic catabolite inactivation in Saccharomyces cerevisiae. Rev Biol Celular 21:305-319.

Hu Z, Chen K, Xia Z, Chavez M, Pal S, Seol JH, Chen CC, Li W and Tyler JK (2014) Nucleosome loss leads to global transcriptional up-regulation and genomic instability during yeast aging. Genes Dev 28:396-408. 
Jiang JC, Jaruga E, Repnevskaya MV and Jazwinski SM (2000) An intervention resembling caloric restriction prolongs life span and retards aging in yeast. FASEB J 14:2135-2137.

Kanehisa M, Araki M, Goto S, Hattori M, Hirakawa M, Itoh M, Katayama T, Kawashima S, Okuda S, Tokimatsu T and Yamanishi Y (2008) KEGG for linking genomes to life and the environment. Nucleic Acids Res 36:D480-D484.

Kato H, Kira S and Kawamukai M (2013) The transcription factors Atf1 and Pcr1 are essential for transcriptional induction of the extracellular maltase Agl1 in fission yeast. PLoS One 8:e80572.

Kelly M, Burke J, Smith M, Klar A and Beach D (1988) Four matingtype genes control sexual differentiation in the fission yeast. EMBO J 7:1537-1547.

Kim DU, Hayles J, Kim D, Wood V, Park HO, Won M, Yoo HS, Duhig T, Nam M, Palmer G et al. (2010) Analysis of a genome-wide set of gene deletions in the fission yeast Schizosaccharomyces pombe. Nat Biotechnol 28:617-623.

Kim HG, Kim BC, Park EH, Ahn K and Lim CJ (2004) Differential regulation of three genes encoding glutathione $\mathrm{S}$-transferases in Schizosaccharomyces pombe. Mol Cells 18:332-339.

Laboucarié T, Detilleux D, Rodriguez-Mias RA, Faux C, Romeo Y, Franz-Wachtel M, Krug K, Maček B, Villén J, Helmlinger D et al. (2017) TORC1 and TORC2 converge to regulate the SAGA co-activator in response to nutrient availability. EMBO Rep 18:2197-2218.

Langmead B, Trapnell C, Pop M and Salzberg SL (2009) Ultrafast and memory-efficient alignment of short DNA sequences to the human genome. Genome Biol 10:R25.

Lee JH, Bassel-Duby R and Olson EN (2014) Heart- and musclederived signaling system dependent on MED13 and Wingless controls obesity in Drosophila. Proc Natl Acad Sci U S A 111:9491-9496.

Lee SJ, Murphy CT and Kenyon C (2009) Glucose shortens the life span of C. elegans by downregulating DAF-16/FOXO activity and aquaporin gene expression. Cell Metab 10:379-391.

Li B and Dewey CN (2011) RSEM: accurate transcript quantification from RNA-Seq data with or without a reference genome. BMC Bioinformatics 12:323.

Li H and Durbin R (2010) Fast and accurate long-read alignment with Burrows-Wheeler transform. Bioinformatics 26:589-595.

Li W, Prazak L, Chatterjee N, Grüninger S, Krug L, Theodorou D, Dubnau J. (2013) Activation of transposable elements during aging and neuronal decline in Drosophila. Nat Neurosci 16:529-531.

Lin SJ, Defossez PA and Guarente L (2000) Requirement of NAD and SIR2 for life-span extension by calorie restriction in Saccharomyces cerevisiae. Science 289:2126-2128.

Liu Z, Li R, Dong Q, Bian L, Li X and Yuan S (2015) Characterization of the non-sexual flocculation of fission yeast cells that results from the deletion of ribosomal protein L32. Yeast 32:439-449.

Ma L, Ho K, Piggott N, Luo Z and Measday V (2012) Interactions between the kinetochore complex and the protein kinase A pathway in Saccharomyces cerevisiae. G3 (Bethesda) 2:831-841.

Mair W, Morantte I, Rodrigues AP, Manning G, Montminy M, Shaw RJ and Dillin A (2011) Lifespan extension induced by AMPK and calcineurin is mediated by CRTC-1 and CREB. Nature 470:404-408.

Malecki M, Bitton DA, Rodríguez-López M, Rallis C, Calavia NG, Smith GC and Bähler J (2016) Functional and regulatory profiling of energy metabolism in fission yeast. Genome Biol 17:24

Mallet PL, Larochelle M and Bachand F (2017) Multiple transcriptional and Post-transcriptional pathways collaborate to control sense and antisense RNAs of Tf2 retroelements in fission yeast. Genetics 205:621-632.
Mata J and Bahler J (2006) Global roles of Ste11p, cell type, and pheromone in the control of gene expression during early sexual differentiation in fission yeast. Proc Natl Acad Sci U S A 103:15517-15522.

Mata J, Wilbrey A and Bahler J (2007) Transcriptional regulatory network for sexual differentiation in fission yeast. Genome Biol 8:R217.

Matangkasombut O, Auty R and Buratowski S (2004) Structure and function of the TFIID complex. Adv Protein Chem 67:67-92.

Matsuzawa T, Fujita Y, Tanaka N, Tohda H, Itadani A and Takegawa K (2011) New insights into galactose metabolism by Schizosaccharomyces pombe: isolation and characterization of a galactose-assimilating mutant. J Biosci Bioeng 111:158-166.

Matsuzawa T, Ohashi T, Hosomi A, Tanaka N, Tohda H and Takegawa K (2010) The gld1+ gene encoding glycerol dehydrogenase is required for glycerol metabolism in Schizosaccharomyces pombe. Appl Microbiol Biotechnol 87:715-727.

Maxwell PH (2016) What might retrotransposons teach us about aging? Curr Genet 62:277-282.

Morrow G, Battistini S, Zhang P and Tanguay RM (2004) Decreased lifespan in the absence of expression of the mitochondrial small heat shock protein Hsp22 in Drosophila. J Biol Chem 279:43382-43385.

Mortuza R, Chen S, Feng B, Sen S and Chakrabarti S (2013) High glucose induced alteration of SIRTs in endothelial cells causes rapid aging in a p300 and FOXO regulated pathway. PLoS One 8:e54514.

Muller S and Almouzni G (2017) Chromatin dynamics during the cell cycle at centromeres. Nat Rev Genet 18:192-208.

Murton HE, Grady PJ, Chan TH, Cam HP and Whitehall SK (2016) Restriction of retrotransposon mobilization in Schizosaccharomyces pombe by transcriptional silencing and higher-order chromatin organization. Genetics 203:1669-1678.

Nehlin JO, Carlberg M and Ronne H (1991) Control of yeast GAL genes by MIG1 repressor: a transcriptional cascade in the glucose response. EMBO J 10:3373-7.

Papadopoli D, Boulay K, Kazak L, Pollak M, Mallette F, Topisirovic I and Hulea L (2019) mTOR as a central regulator of lifespan and aging. F1000Res 8:F100.

Papai G, Frechard A, Kolesnikova O, Crucifix C, Schultz P and BenShem A (2020) Structure of SAGA and mechanism of TBP deposition on gene promoters. Nature 577:711-716.

Rallis C, Codlin S and Bahler J (2013) TORC1 signaling inhibition by rapamycin and caffeine affect lifespan, global gene expression, and cell proliferation of fission yeast. Aging Cell 12:563-573.

Rallis C, Lopez-Maury L, Georgescu T, Pancaldi V and Bahler J (2014) Systematic screen for mutants resistant to TORC1 inhibition in fission yeast reveals genes involved in cellular ageing and growth. Biol Open 3:161-171.

Reverter-Branchat G, Cabiscol E, Tamarit J and Ros J (2004) Oxidative damage to specific proteins in replicative and chronologicalaged Saccharomyces cerevisiae: common targets and prevention by calorie restriction. J Biol Chem 279:31983-31989.

Roux AE, Leroux A, Alaamery MA, Hoffman CS, Chartrand P, Ferbeyre G and Rokeach LA (2009) Pro-aging effects of glucose signaling through a $\mathrm{G}$ protein-coupled glucose receptor in fission yeast. PLoS Genet 5:e1000408.

Saitoh S, Mori A, Uehara L, Masuda F, Soejima S and Yanagida M (2015) Mechanisms of expression and translocation of major fission yeast glucose transporters regulated by CaMKK/ phosphatases, nuclear shuttling, and TOR. Mol Biol Cell 26:373-386.

Sanchez-Perez I, Renwick SJ, Crawley K, Karig I, Buck V, Meadows JC, Franco-Sanchez A, Fleig U, Toda T and Millar JB (2005) The DASH complex and Klp5/Klp6 kinesin coordinate bipolar chromosome attachment in fission yeast. EMBO J 24:2931-2943. 
Santt O, Pfirrmann T, Braun B, Juretschke J, Kimmig P, Scheel H, Hofmann K, Thumm M and Wolf DH (2008) The yeast GID complex, a novel ubiquitin ligase (E3) involved in the regulation of carbohydrate metabolism. Mol Biol Cell 19:3323-3333.

Seike T, Nakamura T and Shimoda C (2013) Distal and proximal actions of peptide pheromone $\mathrm{M}$-factor control different conjugation steps in fission yeast. PLoS One 8:e69491.

Shah SB, Parmiter D, Constantine C, Elizalde P, Naldrett M, Karpova TS and Choy JS (2019) Glucose signaling is connected to chromosome segregation through protein kinase a phosphorylation of the Dam1 kinetochore subunit in Saccharomyces cerevisiae. Genetics 211:531-547.

Sideri T, Rallis C, Bitton DA, Lages BM, Suo F, Rodríguez-López M, Du LL and Bähler J (2014) Parallel profiling of fission yeast deletion mutants for proliferation and for lifespan during long-term quiescence. G3 (Bethesda) 5:145-155.

Sugimoto A, Iino Y, Maeda T, Watanabe Y and Yamamoto M (1991) Schizosaccharomyces pombe ste11+ encodes a transcription factor with an HMG motif that is a critical regulator of sexual development. Genes Dev 5:1990-1999.

Suzuki S, Matsuzawa T, Nukigi Y, Takegawa K and Tanaka N (2010) Characterization of two different types of UDP-glucose/galactose 4-epimerase involved in galactosylation in fission yeast. Microbiology 156:708-718.

Takahashi K, Takayama Y, Masuda F, Kobayashi Y and Saitoh S (2005) Two distinct pathways responsible for the loading of CENP-A to centromeres in the fission yeast cell cycle. Philos Trans R Soc Lond B Biol Sci 360:595-606; discussion 606-607.

Takegawa K., Matsuzawa T. (2014) Insights into metabolism and the galactose recognition system from microarray analysis in the fission yeast Schizosaccharomyces pombe. In: Anazawa $\mathrm{H}$ and Shimizu S (eds) Microbial Production. Springer, Tokyo, pp 109-118.

Tanabe T, Kawamukai M and Matsuo Y (2020) Glucose limitation and pkal deletion rescue aberrant mitotic spindle formation induced by Mal3 overexpression in Schizosaccharomyces pombe. Biosci Biotechnol Biochem 84:1667-1680.

Tanaka N, Ohuchi N, Mukai Y, Osaka Y, Ohtani Y, Tabuchi M, Bhuiyan MS, Fukui H, Harashima S and Takegawa K (1998) Isolation and characterization of an invertase and its repressor genes from Schizosaccharomyces pombe. Biochem Biophys Res Commun 245:246-253.

Tarazona S, Garcia-Alcalde F, Dopazo J, Ferrer A and Conesa A (2011) Differential expression in RNA-seq: a matter of depth. Genome Res 21:2213-2223.

Taricani L, Feilotter HE, Weaver C and Young PG (2001) Expression of hsp16 in response to nucleotide depletion is regulated via the spc1 MAPK pathway in Schizosaccharomyces pombe. Nucleic Acids Res 29:3030-3040.

Trapnell C, Pachter L and Salzberg SL (2009) TopHat: discovering splice junctions with RNA-Seq. Bioinformatics 25:1105-1111.
Valbuena N and Moreno S (2010) TOR and PKA pathways synergize at the level of the Ste11 transcription factor to prevent mating and meiosis in fission yeast. PLoS One 5:e11514.

Vassiliadis D, Wong KH, Andrianopoulos A and Monahan B (2019) A genome-wide analysis of carbon catabolite repression in Schizosaccharomyces pombe. BMC Genomics 20:251.

Veal EA, Toone WM, Jones N and Morgan BA (2002) Distinct roles for glutathione S-transferases in the oxidative stress response in Schizosaccharomyces pombe. J Biol Chem 277:35523-35531.

Walker GA and Lithgow GJ (2003) Lifespan extension in C. elegans by a molecular chaperone dependent upon insulin-like signals. Aging Cell 2:131-139.

Weinberger M, Mesquita A, Caroll T, Marks L, Yang H, Zhang Z, Ludovico P and Burhans WC (2010) Growth signaling promotes chronological aging in budding yeast by inducing superoxide anions that inhibit quiescence. Aging (Albany NY) 2:709-726.

White JW, Swartz FJ and Swartz AF (1985) Excess glucose intake induces accelerated beta-cell polyploidization in normal mice: a possible deleterious effect. J Nutr 115:271-278.

Wierman MB, Maqani N, Strickler E, Li M and Smith JS (2017) Caloric restriction extends yeast chronological life span by optimizing the Snf1 (AMPK) signaling pathway. Mol Cell Biol 37:e00562-16.

Winston F, Durbin KJ and Fink GR (1984) The SPT3 gene is required for normal transcription of Ty elements in $S$. cerevisiae. Cell 39:675-682.

Ye J, Fang L, Zheng H, Zhang Y, Chen J, Zhang Z, Wang J, Li S, Li R, Bolund L and Wang J (2006) WEGO: a web tool for plotting GO annotations. Nucleic Acids Res 34:w293-w297.

Zhang D, Lu H, Chen Z, Wang Y, Lin J, Xu S, Zhang C, Wang B, Yuan Z, Feng X et al. (2017) High glucose induces the aging of mesenchymal stem cells via Akt/mTOR signaling. Mol Med Rep 16:1685-1690.

Zhang S, Chang L, Alfieri C, Zhang Z, Yang J, Maslen S, Skehel $\mathrm{M}$ and Barford D. (2016) Molecular mechanism of APC/C activation by mitotic phosphorylation. Nature 533:260-264.

\section{Supplementary material}

The following online material is available for this article: Table S1 - The quantitative change in the expression of individual genes depending on the glucose concentration.

Associate Editor: Roberto Hirochi Herai

License information: This is an open-access article distributed under the terms of the Creative Commons Attribution License (type CC-BY), which permits unrestricted use, distribution and reproduction in any medium, provided the original article is properly cited.Cupplina 